\title{
Idiopathic multicentric castleman's disease with normal interleukin-6 levels
}

\begin{abstract}
We report a rare case of idiopathic multicentric Castleman's disease with normal interleukin-6 levels. A previously healthy 30 -year old lady presented with a 9-month history of progressive generalised lymphadenopathy associated with fatigue, fevers and night sweats without anorexia or weight loss. Physical examination revealed mobile and non-tender lymphadenopathy in the cervical, axillary and inguinal regions. Laboratory investigations were remarkable for raised inflammatory markers. Thoracic and abdominal CT scan demonstrated multiple enlarged lymph nodes above and below the diaphragm. Results of right axillary lymph node excisional biopsy was consistent with Castleman's disease and bone marrow biopsy performed revealed marrow involvement, leading to a diagnosis of multicentric Castleman's disease. Serology for human immunodeficiency virus and human herpes virus- 8 returned negative, and interleukin-6 levels were normal. The patient was treated with a 3-month course of high dose corticosteroid therapy with resultant disease remission beyond two years follow up.
\end{abstract}

Keywords: lymphadenopathy, excisional biopsy, hypercytokinemia, axilla, hypochromasia
Volume 6 Issue I - 2018

\author{
Ray Mun Koo,' Nalini Patil' \\ 'Department of Clinical Haematology, Canberra Hospital, \\ Australia \\ ${ }^{2}$ Australian National University, Australia
}

Correspondence: Ray Mun Koo, Clinical Haematology Department, Canberra Hospital, Yamba Drive, Garran 2605, ACT Australia, Fax (02) 6174 5544, Tel (02) 6174 85।4, Email RayMun.Koo@act.gov.au

Received: September 05, 2017 | Published: February 06, 2018
Abbreviations: MCD, multicentric castleman's disease; IL6, interleukin-6; HHV-8, human herpes virus-8; ESR, erythrocyte sedimentation rate; CRP, c-reactive protein; HIV, human immunodeficiency; EBV, epstein-barr virus; $\mathrm{UCD}$, unicentric $\mathrm{CD}$; $\mathrm{HV}$, hyaline vascular; $\mathrm{PC}$, plasma cell

\section{Introduction}

Multicentric Castleman's disease (MCD) is a rare systemic lymphoproliferative disorder characterised by polylymphadenopathy, B-symptoms and classical lymph node histological findings. Hypercytokinemia, particularly interleukin-6 (IL-6), secondary to human herpes virus-8 (HHV-8) infection is key to the pathogenesis of MCD. However, MCD can occur independent of HHV-8- an entity known as idiopathic MCD (iMCD). ${ }^{1}$ Uncommonly, a subset of patients can have normal IL-6 levels thus suggesting that other cytokines and pathways contribute to pathogenesis of iMCD.

\section{Case presentation}

A 30-year old lady presented with progressively worsening generalised mobile and non-tender lymphadenopathy over 9-month duration. The lymph nodes were predominantly in her cervical, axillary and inguinal regions; and largest in her right axilla. There was associated fatigue, pyrexia and night sweats without anorexia or weight loss. These symptoms were accompanied by frequent episodes of upper respiratory tract infections and sinusitis requiring oral antibiotic therapy. She had no past medical history of significance and took no regular medications.

On presentation, physical examination revealed normal vital parameters including temperature, blood pressure and oxygen saturation. Lymph node examination revealed bilateral sub-centimetre cervical lymphadenopathy and a single $2 \mathrm{~cm}$ right axillary lymph node. All palpable lymph nodes were soft, mobile and non-tender.
There was no palpable inguinal lymphadenopathy. Abdominal examination identified tender hepatomegaly of $15 \mathrm{~cm}$ and a palpable spleen tip. Neurological, cardiovascular and respiratory examination was normal.

Laboratory investigations showed a normal full blood count apart from mild thrombocytosis, rouleaux formation and hypochromasia in blood film. Inflammatory markers were elevated with an erythrocyte sedimentation rate (ESR) of $75 \mathrm{~mm} / \mathrm{h}$ and C-reactive protein (CRP) of $19.9 \mathrm{mg} / \mathrm{L}$. Liver function test, lactate dehydrogenase level, renal function, angiotensin converting enzyme, C3 and C4 levels were normal. Serological studies for human immunodeficiency (HIV), HHV-8, Epstein-Barr Virus (EBV), Hepatitis B and C infections were negative. IL-6 levels were normal. Serum immunoglobulins revealed elevated $\operatorname{IgG}(36.21 \mathrm{~g} / \mathrm{L})$ and $\operatorname{IgA}(6.78 \mathrm{~g} / \mathrm{L})$ but normal $\operatorname{IgM}$ levels $(2.06 \mathrm{~g} / \mathrm{L})$. Serum $\operatorname{IgG}$ subclasses were elevated, consistent with the known polyclonal hypergammaglobulinemia. Serum electrophoresis was consistent with a polyclonal immune response and immunofixation did not detect a monoclonal protein or light chain. CT of the chest, abdomen and pelvis identified mild submental, submandibular and para-aortic lymphadenopathy with prominent bilateral axillary $(18 \mathrm{~mm})$ and inguinal lymphadenopathy $(14 \mathrm{~mm})$. There was no hilar or mediastinal lymphadenopathy.

Excisional biopsy of right axillary lymph node revealed a dominant interfollicular expansion with increased number of follicles (Figure 1). There was 'onion skin' concentric arrangement of the mantle zone (Figure 2), characterised by a mixture of lymphocytes and plasma cells (Figure 3). No granulomas were seen. Lymphoid follicles and flow cytometry were normal and immunohistochemical staining for HHV8 was negative. Bacterial, fungal culture and prolonged mycobacterial culture were also negative.

Bone marrow biopsy was performed revealing a mildly hypercellular marrow aspirate with a prominent pleomorphic 
plasmacytosis, reactive lymphocytes, reactive monocytosis and increased macrophage demonstrating hemophagocytosis. Trephine biopsy show preserved trilineage haemopoiesis and architecture with moderate sized aggregates of plasma cells throughout the marrow in a perivascular distribution, which stain strongly for CD38 and mildly for CD138. Marrow immunophenotyping did not reveal any abnormal features.

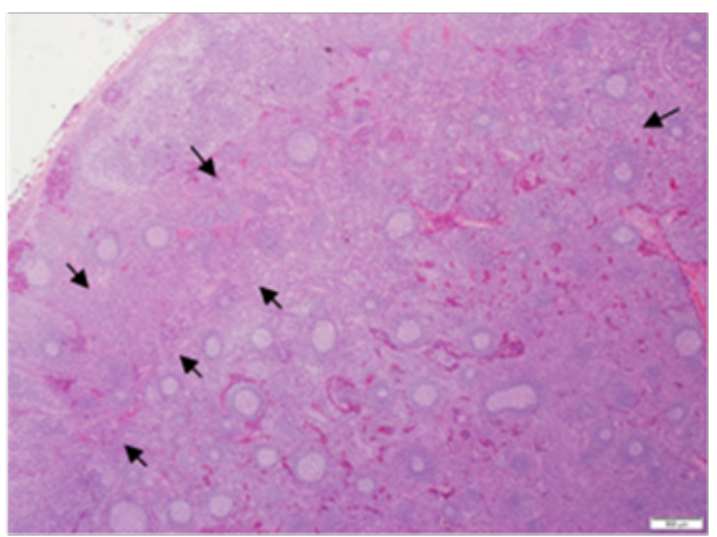

Figure I Field of view at low power magnification revealing dominant interfollicular expansion (black arrows).

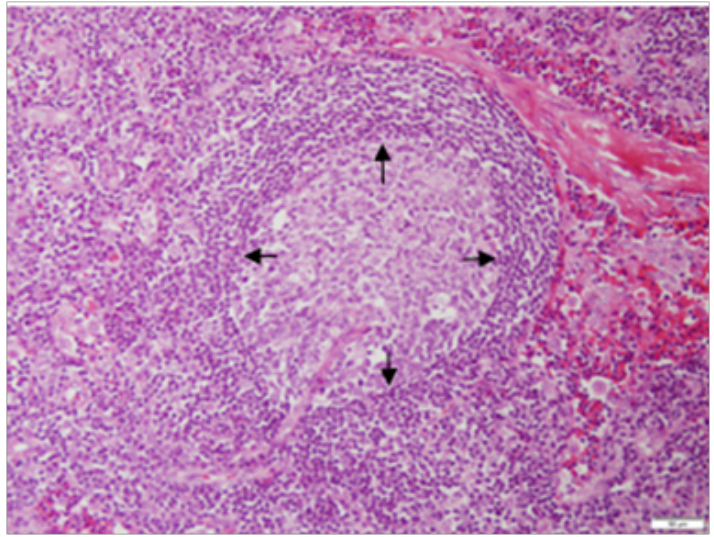

Figure 2 Concentric ring of small lymphocytes surrounding a hyalinised vessel in an "onion skin" arrangement (black arrows).

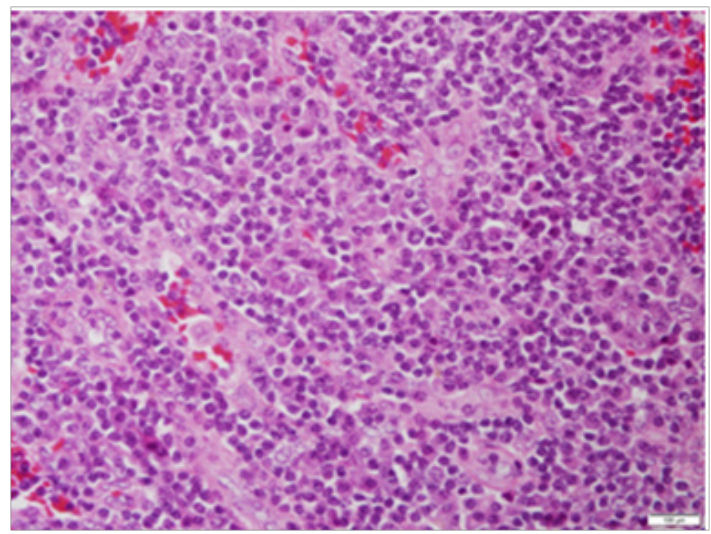

Figure 3 Population of plasma cells seen in the sampled lymph node.

A diagnosis of idiopathic MCD with bone marrow involvement was made. Our patient was treated with Prednisolone $75 \mathrm{mg}(1 \mathrm{mg} /$ $\mathrm{kg}$ ) daily over a 2-week duration, which was then decreased to $50 \mathrm{mg}$ daily for another 2 weeks. Prednisolone therapy was then tapered off completely over the following 2 months. There was significant reduction in lymphadenopathy and resolution of B-symptoms with oral corticosteroid therapy. Over the following 2years, our patient's symptoms were well controlled with no worsening lymphadenopathy despite cessation of oral corticosteroid therapy. Repeat bone marrow biopsy performed showed non-specific reactive changes and absence of direct marrow involvement with Castleman's disease. She remains in remission and is currently receiving 3-monthly reviews in our clinic.

\section{Discussion}

Castleman's disease (CD) is a rare group of heterogeneous lymphoproliferative disorders characterised by nodal expansion with partial preservation of underlying lymph node structure secondary to hypercytokinemia, particularly IL-6. CD, also known as angiofollicular lymph node hyperplasia, was first described by Benjamin Castleman in 1954 who subsequently described a cohort of 13 patients with localised, solitary hyperplastic mediastinal lymphadenopathy containing small, hyalinised follicles with marked interfollicular vascular proliferation., ${ }^{2,3}$

Multicentric CD (MCD) is used to describe CD with diffuse lymphadenopathy, while unicentric $\mathrm{CD}$ (UCD) has localised disease involvement. $\mathrm{CD}$ can also be classified histopathologically into 4 variants: hyaline vascular (HV), plasma cell (PC), mixed and plasmablastic ${ }^{4}$ PC variant is seen in $80-90 \%$ of MCD and histologically appears as intense plasmacytosis in the interfollicular areas of the nodes with prominent increase in capillaries and postcapillary venules. HV is seen more commonly in UCD but also in $10 \%$ of MCD and is characterised by a widened mantle zone composed of concentric ring of small lymphocytes with hyalinised vessels in an "onion skin" arrangement."

MCD is more common in males and often presents in the 6th decade of life although earlier presentations are not uncommon. Clinical presentation ranges from fluctuating multi-regional lymphadenopathy associated with fevers, weight loss and anorexia to more severe systemic inflammatory responses, organomegaly and even death. ${ }^{5-7}$ Laboratory investigations often reveal anaemia, thrombocytosis, raised ESR, hypoalbuminemia and hypergammaglobulinemia. Cytokines, notably IL-6 and IL-10, are elevated but normal IL-6 levels are seen in approximately $10 \%$ of cases. ${ }^{8}$ Bone marrow biopsy typically shows intense polyclonal plasmacytosis. Imaging in the form of CT demonstrate involvement of multiple lymph node groups which show low-FDG uptake on positron emission tomography.

MCD is commonly associated with HHV-8 and HIV infection. The pathogenesis of MCD remains poorly understood but has been associated with excessive IL-6 or related polypeptide release from HHV-8 infection. Up to a third of patients with MCD do not have HHV-8 and are known as idiopathic MCD (iMCD). ${ }^{1,4}$ The pathogenesis of iMCD is less well known and is suggested to involve immune dysregulation with a common pathway leading to elevated proinflammatory cytokine release leading to iMCD symptomatology and lymph node involution. ${ }^{4}$

MCD diagnosis is made clinicopathological with confirmation on classical features on lymph node biopsy in conjunction with symptoms of active disease. High-dose corticosteroid therapy is firstline treatment for $\mathrm{MMCD}$ which invariably lead to relapse, requiring addition of other immunosuppressive or cytotoxic lymphoma-based chemotherapy. ${ }^{8}$ Recently, biological anti-IL-6 agents in the form of tocilizumab and siltuximab have both been proven to be effective 
in the treatment of $\mathrm{MCD}^{9,10}$ with siltuximab been approved for the treatment of iMCD in the United States.

The clinical course can range between states of indolence to a rapidly progressive form in immuno-compromised individuals leading to death in weeks. A systematic review by Liu et al described a 2 -year survival of $88 \%$ and $22 \%$ patient mortality by the end of a 29 -month median follow-up period. ${ }^{8}$ There was also an increased prevalence of solid organ and haematological malignancy in iMCD patients which correlates with a poorer prognosis.

\section{Conclusion}

Multicentric Castleman's disease is a rare lymphoproliferative disorder closely associated with human herpes virus- 8 infection that is characterised by generalised lymphadenopathy, B-symptoms and classical lymph node histological features. Disease pathogenesis is poorly understood but is associated with hypercytokinemia, particularly interleukin-6, from human herpes virus- 8 infection. A third of patients does not have human herpes virus- 8 infection and is known as idiopathic multicentric Castleman's disease. Only $10 \%$ of these patients have normal interleukin-6 levels. Treatment option includes corticosteroids, immunosuppressive therapy, cytotoxic chemotherapy or biological anti-interleukin 6 agents. Clinical course can range from a state of indolence to rapidly progressive disease. The 2 year-survival is described at $88 \%$.

\section{Acknowledgements}

The authors of this article acknowledge the contribution of Dr Tamara Welham, Pathology Registrar at the Canberra Hospital, Australia for obtaining the histopathology slides for this article.

\section{Conflict of interest}

The authors declare that they have no conflicts of interest.

\section{Consent}

Written and verbal informed consent was obtained from the patient for publication of this case report.

\section{References}

1. Barozzi P, Luppi M, Masini L, et al. Lymphotropic herpes virus (EBV, HHV-6, HHV-8) DNA sequences in HIV negative Castleman's disease. Clin Mol Pathol. 1996;49(4):M232-M235.

2. Castleman B, Iverson L, Menendez VP. Localised mediastinal lymph node hyperplasia resembling thymoma. Cancer. 1956;9(4):822-830.

3. Castleman B, Towne VW. Case records of the Massachusetts General Hospital; weekly clinicopathological exercises; founded by Richard C. Cabot. N Engl J Med. 1954;251(10):396-400.

4. Fajgenbaum DC, van Rhee F, Nabel CS. HHV-8-negative, idiopathic multicentric Castleman disease: novel insights into biology, pathogenesis and therapy. Blood. 2014;123(19):2924-2933.

5. Waterston A, Bower M. Fifty years of multicentric Castleman's disease. Acta Oncol. 2004;43(8):698-704.

6. Soumerai JD, Sohani AR, Abramson JS. Diagnosis and management of Castleman disease. Cancer Control. 2014;21(4):266-278.

7. Dispenzieri A, Armitage JO, Loe MJ, et al. The clinical spectrum of Castleman's disease. Am J Hematol. 2012;87(11):997-1002.

8. Liu AY, Nabel CS, Finkelman BS, et al. Idiopathic multicentric Castleman's disease: a systematic literature review. Lancet Haematol. 2016;3(4):e163-e175.

9. Van Rhee F, Wong RS, Munshi N, et al. Situximab for multicentric Castleman's disease: a randomised, double-blind, placebo-controlled trial. Lancet Oncol. 2014;15(9):966-974.

10. Nishimoto N, Kanakura Y, Aozasa K, et al. Humanized anti-interleukin-6 receptor antibody treatment of multicentric Castleman disease. Blood. 2005;106(8):2627-2632. 